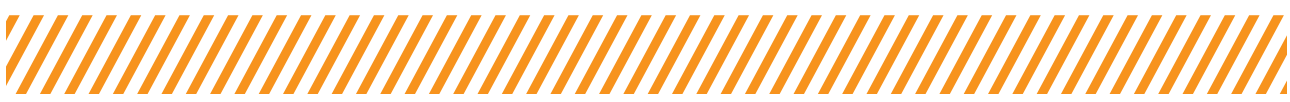

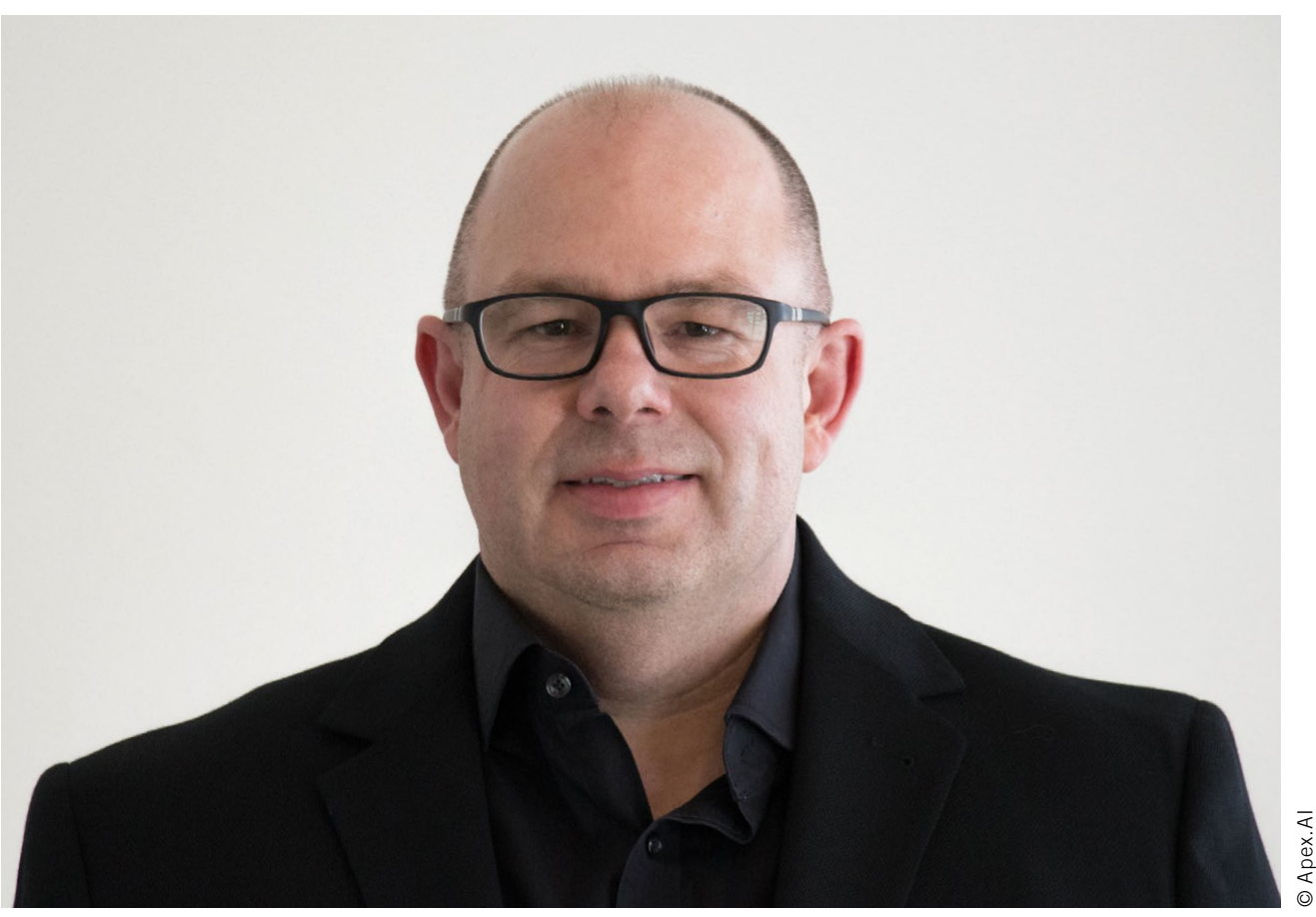

Dr. Jan Becker CEO of Apex.Al in Palo Alto (USA)

\section{Standards for Automotive Operating Systems}

Although the German automotive industry is discussing a common operating system, it seems that every stakeholder wants their proprietary solution to prevail. This is steering us toward a situation that we have already experienced: that of the smartphone. Twelve years ago, the market for smartphone operating systems was fragmented and consisted of Apple iOS and half a dozen other systems. Today, Android has a 72-\% market share and iOS the remaining $27 \%$. The reason for this is simple:

Operating system markets are winner-take-all markets.

Of course, every large player in the automotive world wants to be like Apple and "own an (i)OS." This opportunity really did exist eight years ago; Tesla grasped it. Time has run out for all the others. What is more, the fact that a software development culture cannot be established overnight has escaped attention and that such a transformation requires a huge budget. A budget that Apple and Tesla have available, but not necessarily the traditional automotive manufacturers. However, culture and money only represent two of the hurdles; the third is the lack of outstanding software architects. They are a rare species that seldom wander into the automotive domain. And if the carmakers do manage to find software developers, then intensive training is required to make them familiar with in-house systems.

Which leaves us with the Android model. In this case, interfaces are open, and the source code is freely available. However, this common and open basis cannot originate from one of the large players because this would mean that the base layer would not be widely accepted. In the ideal case, this basis would already enjoy widespread distribution to prevent being too late. And if this system would then also be used internationally in universities, then future software developers would already be familiar with it before they take up their first jobs. Is this too good to be true?

Perhaps not. A glance at the robotics sector shows us that it is exactly these challenges that have been solved with the open-source Robot Operating System (ROS). The more robust second release (ROS 2) has been available for four years and, in the past few months, has been supplemented by the safetycertified version (ISO 26262 ASIL D).

A further challenge for OEMs and tier-1 suppliers is the productivity level of software development: Automotive projects often employ hundreds of software developers. The enormous level of adaption effort required can be avoided if a truly consistent software architecture is created instead of combining awkward legacy components with new software. Revisiting the smartphone analogy: No one would install the current iPhone 13 software and attempt to combine it with software developed for a Nokia telephone from 2002 and a Blackberry from 2006.

Perhaps the sector should take the path Android trod: Use a common base layer and concentrate on the most important aspects, namely the features that differentiate in terms of customers and competitors. The resulting large volume of new features means that there is more than enough work for OEM and tier-1 software developers to do. 